\title{
LA PUNTUACIÓN Y LAS UNIDADES TEXTUALES: UNA PERSPECTIVA DISCURSIVA PARA EL ESTUDIO DE LOS PROBLEMAS DE SU USO Y PARA SU ENSEÑANZA
}

\author{
Carlos Sánchez Avendaño \\ Investigador del INIL (Instituto de Investigaciones Lingüisticas)
}

Recibido 26-IV-2004 • Aceptado 28-IV-2004

"La puntuación es como un termómetro de la escritura".

Daniel Cassandy (1955)

Resumen: En este artículo, se realiza un análisis pormenorizado de los textos para la enseñanza de la puntuación en Costa Rica y del uso de los signos de puntuación por parte de estudiantes de primer ingreso a la universidad desde una perspectiva discursiva.

Palabras clave: Expresión Escrita, Signos de Puntuación, Unidades Textuales, Perspectiva Discursiva de la Puntuación.

\begin{abstract}
This article presents a detailed analysis concerning textbooks for the teaching of punctuation as well as punctuation marks used by university students. The analysis is carried out within a discursive perspective.
\end{abstract}

Key words: Writing, Punctuation Marks, Textual Units, Discursive Perspective of Punctuation.

\section{Introducción}

Por lo regular, se da por sentado que el estudio de los signos de puntuación pertenece al ámbito de un curso de redacción, quizás porque se considera que estos elementos son uno de los pilares de la expresión escrita. A pesar de que estos signos se estudian desde los primeros años de escolarización, es común escuchar en nuestro medio que los estudiantes presentan una puntuación simplemente "pavorosa" (además de una pésima ortografía y, en general, un manejo deficiente del registro escrito). Estas aseveraciones pertenecen a la esfera de la impresión y, aunque ameritarían un estudio serio al respecto, se ha convertido en un lugar común de parte de los educadores el quejarse sobre el problema sin ahondar en las causas de este y, como corolario, en sus posibles soluciones.

Como se dijo, si el deficiente manejo de la puntuación por parte de los estudiantes 
costarricenses ha alcanzado niveles "escandalizadores", ¿qué han sugerido las investigaciones al respecto?, ¿en qué consiste el problema?, ¿cómo solucionarlo? El presente trabajo tiene como objetivo central arrojar alguna luz en torno a este componente de la expresión escrita.

\section{Los textos para la enseñanza de la puntuación en Costa Rica}

\subsection{La presentación de los signos de puntuación y su tratamiento}

A pesar del papel central que la puntuación reviste en el registro escrito como mecanismo distributivo de la información, no todos los libros de texto para la enseñanza de la redacción publicados en Costa Rica han abordado el tema. Por lo regular, la puntuación ocupa un capítulo o una sección en este tipo de textos, en los que se explican en forma de listas los usos de los signos de puntuación en forma totalmente desligada del hilo expositivo general del resto del libro; en otras palabras, no se evidencia la relación de la puntuación con la segmentación informativa dentro del texto como totalidad o sus componentes (párrafos y enunciados), no se liga el tema con el uso del vocabulario (por ejemplo, como estrategia para evitar la repetición de palabras por medio de la elipsis y el punto y coma o la coma como marcadores de eliminación de un elemento repetido) ni de la gramática (como el uso del punto y coma antes de un conector, o el de la coma para separar incisos textuales).

En general, los textos presentan el nombre de cada signo seguido de una enumeración de usos muy concretos y sin ningún orden expositivo en relación con su papel dentro del texto como marcador de una unidad textual; además, la presentación del tema se hace como parte de la sección de ortografía, con la cual guarda poco en común ${ }^{1}$. Esta es el tratamiento que recibe este tema en Soto Alfaro et al. (1983) $)^{2}$, Garita y Quesada $(1988)^{3}$, Sánchez y Vargas (1990), Bolaños (1996), Müller Delgado (1996) ${ }^{4}$ y Pacheco y Álvarez (1997). En Sandino y Varela (1993) se presentan los signos en el capítulo correspondiente a los párrafos, pero como simple disposición del libro, pues no se hace una verdadera trabazón expositiva ni didáctica entre un tema y el otro. En cambio, en Brenes (1990) y Quijano López (1997), la relación entre los signos de puntuación y los párrafos sí es patente, pues se exponen como elementos de segmentación y estructuración del mensaje. Por ello mismo, los autores solamente incluyen la explicación del punto, la coma y el punto y coma (además del guión, en el caso de Quijano); es decir, los signos que se emplean con una función segmentadora.

Otros libros de texto publicados en Costa Rica incluyen el tema distribuido a lo largo de distintos capítulos. Podría pensarse que, debido a esto, se presentan ligados al tema tratado en cada sección; sin embargo, no es así en absoluto. Por lo regular, se exponen distintos usos particulares de cada signo dentro de un capítulo concreto como simple recurso para dosificar su presentación: Sandoval (1970), Sandoval (1978), Vargas y Sandoval (1978abc), Durán y Jiménez (1989).

Otros autores mencionan distintos usos o problemas de puntación en el análisis de ejemplos de redacciones (Bustos, 1983) o en algún aspecto muy concreto de la expresión escrita. Este último sería el caso de Jézer González (1986), quien trata la inserción de elementos explicativos en la oración y su respectiva puntuación, pues asevera que es este un problema común incluso en los autores experimentados.

Por último, tendríamos los textos publicados específicamente para la enseñanza de la puntuación. Al igual que sucede en la enseñanza de la redacción en general (cfs. Sánchez Avendaño), Moisés Vicenzi es el primer pedagogo -del que tengamos 
noticia- que se ocupa del asunto en Costa Rica, en su libro La enseñanza de la puntuación de 1939. Para Vicenzi, la puntuación es un recurso técnico con cuatro aristas: el aspecto formalista dentro de la gramática, el aspecto psicológico, el lógico y el estético. En sus palabras, antes de la enseñanza de la puntuación, debe darse un "proceso psicológico preparatorio, para mostrar al alumno el valor individual e interno, de cada signo" (Vicenzi, 1939:10). También debe ser previa la enseñanza del ordenamiento de las ideas, con criterios de claridad y precisión, para luego poder dominar los signos de puntuación; esta sería la faceta lógica del asunto. Por último, la estética estaría evidenciada por lo monótono que resulta un texto con escasa puntuación, frente a la riqueza ideológica (patente en el uso de los signos) que se encuentra en un escritor experto.

Finalmente, Vicenzi arremete contra el método analítico con el que se enseña la puntuación y la gramática: "Se presenta el problema de la coma, del punto y coma, de los dos puntos y se explica y resuelve sin motivarlo. De esta manera, el alumno lo recibe fríamente. Es que se ha olvidado tanto el aspecto lógico como el estético, a fin de cultivar el aprendizaje en el formal exclusivo. Puede afirmarse que el fracaso apreciado por todos en este ejercicio, se debe, por eso, a la exclusividad del método analítico" (Vicenzi, 1939:13). En su lugar, propone el método sintético ${ }^{5}$ : presentar textos literarios como modelos en los que aparezcan bien empleados los signos que se van a enseñar, con el fin de presentar el tema de forma funcional al estudiante y de incorporar los aspectos formales, lógicos y estéticos ligados a este ${ }^{6}$. Paso seguido, el maestro debe indicarles a los alumnos que presten especial atención al signo por estudiarse durante su lectura de los textos seleccionados; luego, deben explicar su uso y, por último, el profesor debe dirigir las observaciones de estos para conseguir una definición colectiva del uso. El método de Vicenzi incluye la recomendación de que los estudiantes busquen, como tarea, textos escogidos por ellos en los que se utilicen los signos estudiados.

González Picado (1970) también recurre a trozos literarios para ejemplificar los usos de los signos, pero su método es, contrario al de Vicenzi, totalmente deductivo: se expone la regla, se dan ejemplos al respecto y se presentan prácticas en las que se deben colocar los signos pertinentes.

La Puntuación al alcance de todos de Bustos Arratia (1994), editado por primera vez en Chile en 1972 y varias veces reeditado por la Editorial EUNED en Costa Rica desde 1981, es un buen ejemplo del tratamiento exhaustivo del tema en forma de largas listas de usos, sin ningún hilo conceptual que muestre la función textual cohesiva de estos elementos. Bustos Arratia defiende este método didáctico: "Nosotros creemos en las reglas de puntuación, ya que ellas prestan enorme utilidad a la gran masa de personas que carecen de intuición idiomática, y ayudan a resolver dudas a quienes, teniéndola, no poseen un conocimiento científico y organizado de los 'mecanismos' del idioma" (Bustos, 1994:24). Finalmente, propone que la causa de los problemas de puntuación reside en el desconocimiento de la gramática, por lo que trata extensamente nociones de gramática normativa tradicional en algunas de las reglas para el uso de los signos. Con ello, se hace manifiesta la idea de que el manejo de la taxonomía y de la nomenclatura gramaticales tradicionales son indispensables para poder redactar correctamente (cfs. Sánchez Avendaño). En Pazos (1990) resulta aún más evidente la ausencia de este marco conceptual de fondo que explique la razón de ser de estos signos convencionales, pues la autora se limita a presentar largas listas de usos para cada uno.

\subsection{La concepción sobre el uso y la función de los signos}

Como se puede notar, la concepción sobre el uso y la función de los signos se torna bastante endeble al carecer la exposición 
de una teoría bien planteada sobre su valor textual. La idea de que la puntuación cumple un papel arquitectónico en la estructuración de los textos, formulada ya desde 1930 por Moisés Vicenzi en Costa Rica, no ha sido bien explotada por los autores que han publicado libros para su enseñanza.

Como se verá más adelante, de acuerdo con Figueras (2001), uno de los principales problemas en torno a la puntuación radica en la idea altamente extendida, pero errónea, de que los signos cumplen la función de representar la entonación y las pausas propias del registro oral.

En nuestro medio, esta concepción es la favorita de los autores, quienes consignan este papel como el principal ${ }^{7}$. Así, Bustos Arratia (1994) asegura que los signos de puntuación son "sólo aquellos que constituyen la representación gráfica de las pausas que deben hacerse en una lectura en voz alta" (p. 13), definición que, de acuerdo con esta autora, incluiría a los siguientes signos: la coma, el punto y coma, los dos puntos, el punto (seguido y aparte), los puntos suspensivos, el paréntesis y el guión. Los demás signos, los "auxiliares", serían: las comillas, la diéresis, el apóstrofo, el párrafo (§), el asterisco, la llave o corchete, la manecilla y la raya oblicua. Por último, estarían los signos de entonación: la interrogación y la exclamación.

Bustos Arratia rescata la noción de que los signos son marcas para dotar de claridad el pensamiento plasmado por escrito, tal como lo hacen las pausas en la lectura. Esto lo ejemplifica con el siguiente caso: "Juan come rápido" y “Juan, come rápido" se diferencian en que la primera es una aseveración, mientras que la segunda -marcada mediante una coma que representa una pausa- es una petición u orden. Debemos aclarar que, en este caso en particular, es patente la confusión entre el papel de los signos como recursos del registro escrito y los elementos propios de la oralidad: el uso de la coma en el segundo ejemplo separa un vocativo, pero no señala necesariamente una pausa. De hecho, lo común sería que, para indicar la función vocativa en el registro oral espontáneo, se recurra a la pronunciación del vocablo 'Juan' con una curva de entonación descendente, y no obligatoriamente con una pausa (Cfs. Quilis, 1990).

La noción de los signos como marcadores de pausas se halla, más o menos desarrollada o esbozada, en Sánchez y Vargas (1990), quienes exponen claramente la idea de que algunos signos señalan pausas con duraciones distintas (la coma indica pausas breves; el punto y coma, pausas menores que las del punto y mayores que las de la coma). También es clara esta idea en González Picado (1970) Sandino y Varela (1993), Bolaños (1996), Müller (1996) y Soto Alfaro (1983). Por su parte, Pazos (1990) y Pacheco y Álvarez (1997) ni siquiera mencionan la función general que tendrían los signos, sino que simplemente presentan los listados de usos para cada uno. Brenes (1990) se limita a señalar la función segmentadora con fines de claridad expresiva y precisión en relación con la intención del escritor, aspecto que también mencionan Sánchez y Vargas (1990) y Bustos Arratia (1994). Durán y Jiménez (1989) establecen una relación más estrecha entre la puntuación y la entonación; así, el punto indica un descenso tonal, la coma señala un breve ascenso o descenso de tono, el punto y coma representa un descenso de tono mayor que el de la coma.

Nuevamente, López Quijano sería la excepción a esta tendencia, pues en él se halla casi totalmente ausente la función respiratoria. Para este autor, "los signos de puntuación juegan un papel similar a las señales de tránsito; éstas regulan el movimiento de los vehículos; los signos de puntuación, el orden e importancia de las ideas. Unos harán deternerse al lector, otros le destacarán algo" (Quijano 1997:167).

En general, entonces, se les atribuye a los signos de puntuación el servir como marcas de la respiración. Aunque es cierto que algunos autores recogen también las 
funciones sintácticas y psicológicas de estos, normalmente las supeditan a esta función considerada como "primaria". Como consecuencia, no se encuentra una relación directa entre la puntuación y la segmentación de los textos en unidades que lo componen.

Parece ser que únicamente Moisés Vicenzi (1939) y Quijano López (1997) se escapan de esta tendencia. En el caso particular del primero, es el único autor que ha propuesto una metodogía en la que se privilegian otros aspectos (en sus palabras: el lógico, el psicológico y el estético) por sobre una supuesta necesidad de indicar las pausas en la lectura, aunque tampoco él se escapa de consignar esta función.

\section{Pragmática de la puntuación}

Carolina Figueras publicó, en el 2001, uno de los trabajos más completos sobre el empleo de los signos de puntuación como recursos propios del registro escrito. En su Pragmática de la puntuación, Figueras realiza un análisis pormenorizado de cada signo en forma individual, pero, sobre todo, en su relación con el conjunto del texto; en otras palabras, se trata de abordar la puntuación como fenómeno textual de articulación semántica, como estrategia de cohesión discursiva ${ }^{8}$. Precisamente, Figueras critica la postura tradicional de tratar la puntuación como parte de la ortografía, debido a que estos dos códigos son de naturaleza distinta: los signos de puntuación guardan relación con la construcción del texto; la ortografía, por su parte, es una convención de aplicación regular y no afecta la concatenación de las diversas unidades discursivas.

Para Figueras, el uso apropiado de los signos de puntuación indica madurez en la adquisición del registro escrito. Así, en el caso de los estudiantes, su insuficiente desarrollo de la competencia discursiva explicaría el manejo deficiente que estos muestran de los signos. Del mismo modo, problemas en la comprensión de la estructura sintáctica del texto conllevan a una posible puntuación inadecuada.

La función primordial de los signos de puntuación consiste en segmentar los bloques informativos que conforman el texto, con lo cual se convierten en verdaderos guías y facilitadores de la comprensión del discurso en su totalidad. Aquí residiría la ineficacia de los métodos educativos tradicionales en cuanto a la expresión escrita y, más específicamente, en relación con la didáctica de la puntuación: enumerar los usos normativos de cada signo (tal como lo hacen los autores costarricenses reseñados) sin indicar la función textual y, por consiguiente, su razón de ser fundamental: segmentar en unidades, controlar la interpretación de los escritos y guiar su comprensión.

Para Figueras, resultaría mucho más provechoso para la didáctica de la redacción ayudarles a los estudiantes a descubrir la función jerarquizadora de la información que poseen los signos, e incentivar así un aprendizaje de su empleo como recursos de indicación intencional de cómo debe entenderse lo escrito (de aquí su enfoque pragmático); es decir, enseñarlos como una herramienta eficaz para la expresión escrita. Por el contrario, la idea de que la puntuación constituye un sistema de marcas para que el lector de un texto pueda hacer una pausa y respirar puede llevar a confusión y error en el caso de los aprendices del registro escrito. Si bien es cierto que estos signos nacieron con la función de indicadores declamativos, su papel ha cambiado radicalmente a partir del Renacimiento, cuando se convierten en marcas textuales. Sin embargo, la mayoría de los manuales de puntuación siguen señalando el uso de algunos signos en función de la duración de las pausas propias de la producción oral; es decir, tal como lo indican Sánchez y Vargas (1990) para el punto y el punto y coma y la coma: una pausa de menor longitud conforme se avanza desde el punto hasta la coma. 
En contraste con la indefinición o poca claridad con que la mayoría de los autores tratan el tema, Figueras parte de un principio general, en total armonía con su planteamiento de base discursiva: "La puntuación es un sistema de signos gráficos cuya función es articular y distribuir la información en el texto. Las marcas de puntuación delimitan las distintas unidades lingüísticas que conforman el discurso escrito. Así, el punto final establece el límite de la unidad texto; el punto y aparte define la unidad párrafo; el punto y seguido, la unidad enunciado, etc." (Figueras 2001:15). Entonces, lo que subyace al empleo de los signos no es una relación primigenia con la entonación, sino su valor demarcativo de tipo sintáctico y discursivo en el registro escrito. Esta misma posición se deja ver en otros autores como Cassany (1995) y Ávila (2001).

La consecuencia que señala Figueras de esta confusión teórica no puede ser más elocuente: "El resultado de combinar estas dos teorías de la puntuación -la referida a la lectura y la orientada hacia la escritura- es que, al final, el estudiante acaba por no aprender cuáles son las verdaderas funciones de los signos de puntuación. Y acaba puntuando allí donde le parece que el lector debe pararse a respirar" (Figueras 2001:22).

La función discursiva del sistema de signos de puntuación se revela en su papel segmentador de las unidades textuales, su distribución informativa jerárquica y la indicación del grado de relación que existe entre ellas. Es a partir de esta función que se justificaría una tipología de los signos, tal como la presenta Figueras: el punto final, el punto y aparte, el punto y seguido, el punto y coma, los dos puntos y la coma cumplen la función de determinar las unidades básicas de procesamiento del texto; por ello mismo, serían los signos básicos o de primer régimen. Dentro de estos, el punto y la coma serían las marcas primarias, las propias de una puntuación mínima.
Cuadro 1

Primer régimen de puntuación (Figueras 2001:35)

\begin{tabular}{|l|l|c|}
\hline MARCADOR & $\begin{array}{l}\text { UNIDAD } \\
\text { DELIMITADA }\end{array}$ & \\
\hline Coma & $\begin{array}{l}\text { Sintagma } \\
\text { Enunciados } \\
\text { oracionales } \\
\text { Cláusula } \\
\text { textual } \\
\text { Enunciado } \\
\text { textual }\end{array}$ & $\begin{array}{l}\text { Nivel } \\
\text { microes- } \\
\text { tructural }\end{array}$ \\
Punto y seguido & $\begin{array}{l}\text { Párrafo } \\
\text { Texto }\end{array}$ & $\begin{array}{c}\text { Nivel } \\
\text { macroes- } \\
\text { tructural }\end{array}$ \\
\hline $\begin{array}{l}\text { Punto y aparte } \\
\text { Punto final }\end{array}$ & & \\
\hline
\end{tabular}

En este primer régimen, se encuentran dos tipos de signos: los que funcionan en un nivel horizontal (delimitan unidades contiguas, por debajo del párrafo, que deben interpretarse en relación con el segmento anterior: nivel microestructural) y los que lo hacen en un nivel vertical (organizan y distribuyen del tema general y subtemas o cierran la unidad textual en su totalidad: nivel macroestructural). El punto y seguido, aunque pertenece al micronivel, en realidad sirve de enlace entre los dos niveles.

Existen otras marcas cuya función primordial reside en introducir información suplementaria, por lo cual serían elementos de segundo nivel o de segundo régimen: los guiones largos, los paréntesis y las comillas. Dentro de este segundo régimen tienen cabida otros elementos, más bien indicadores de modalidad y no delimitadores de un segundo discurso: los signos de interrogación y de exclamación y los puntos suspensivos.

\section{Cuadro 2}

Segundo régimen de puntuación (Figueras 2001:36)

\begin{tabular}{|l|l|}
\hline $\begin{array}{l}\text { Introducción de un } \\
\text { segundo discurso }\end{array}$ & $\begin{array}{l}\text { Marcadores de } \\
\text { modalidad }\end{array}$ \\
\hline $\begin{array}{l}\text { Guiones largos } \\
\text { Paréntesis } \\
\text { Comillas }\end{array}$ & $\begin{array}{l}\text { Signos de interrogación } \\
\text { Signos de exclamación } \\
\text { Puntos suspensivos }\end{array}$ \\
\hline
\end{tabular}


El empleo de los signos de segundo régimen implica una mayor complejidad en el texto, en especial cuando incorporan un segundo discurso. Por esta razón, se dice que requieren de un manejo adecuado de la sintaxis, pues el escritor debe ser capaz de insertar un inciso y retomar el curso de su línea expositiva o argumentativa apropiadamente. Precisamente, el manejo inadecuado de los incisos es una de las características principales de los escritores inexpertos.

En todo caso, los signos de primer régimen constituyen un recurso primordial para la segmentación de las unidades informativas y la indicación interpretativa que debe dar el autor, tal como lo resume Figueras en el siguiente cuadro:

\section{Estado de la cuestión: Los problemas de puntuación y segmentación en unidades textuales de los estudiantes costarricenses}

El estado de la cuestión acerca de los problemas en el uso de los signos de puntuación por parte de los alumnos costarricenses no parece definir claramente las características específicas del asunto, no porque no se hayan llevado a cabo investigaciones al respecto, sino por la brevedad y la poca profundidad con las que los diversos autores exponen sus resultados acerca de este tema en especial y, en consecuencia, lo exiguas que resultan las conclusiones.

Cuadro 3

Signos lógicos o de primer régimen (Figueras 2001:46)

\begin{tabular}{|c|c|c|}
\hline & $\begin{array}{l}\text { UNIDAD TEXTUAL } \\
\text { DELIMITADA }\end{array}$ & SIGNIFICADO ASOCIADO \\
\hline \multirow{2}{*}{ 氞 } & $\begin{array}{l}\text { Texto } \\
\text { (signo: punto final) }\end{array}$ & $\begin{array}{l}\text { Unidad comunicativa básica, definida por su complitud } \\
\text { semántica y por su coherencia y relevancia interna. } \\
\text { El hablante explica algo, argumenta a favor de algo, } \\
\text { describe algo, ordena algo, solicita algo, etc. El texto } \\
\text { desarrolla un tema global. }\end{array}$ \\
\hline & $\begin{array}{l}\text { Párrafo } \\
\text { (signo: punto y aparte) }\end{array}$ & $\begin{array}{l}\text { Cada uno de los subtemas individuales que conforman } \\
\text { el contenido global del texto. Los párrafos son las } \\
\text { unidades temáticas en las que se divide el tema } \\
\text { general del que trata el texto. }\end{array}$ \\
\hline \multirow{4}{*}{ 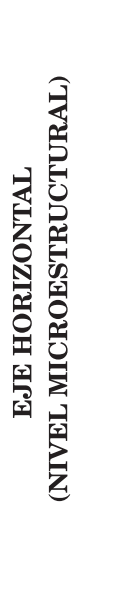 } & $\begin{array}{l}\text { Enunciado textual } \\
\text { (signo: punto y seguido) }\end{array}$ & $\begin{array}{l}\text { Idea, pensamiento, argumento o conclusión parcial, } \\
\text { evento o suceso individual, operación (simple o } \\
\text { compleja), exposición de un dato o un conjunto de } \\
\text { datos relacionados, descripción/ exposición de un } \\
\text { aspecto específico del subtema del párrafo. }\end{array}$ \\
\hline & $\begin{array}{l}\text { Cláusula textual } \\
\text { (signo: punto y coma) }\end{array}$ & $\begin{array}{l}\text { Operación básica que forma parte de una operación } \\
\text { más compleja (descrita en el enunciado); suceso simple } \\
\text { de un evento; parte de un argumento complejo. El } \\
\text { significado de la cláusula depende del significado de } \\
\text { la(s) otra(s) cláusula(s) que forma(n) el enunciado textual. }\end{array}$ \\
\hline & $\begin{array}{l}\text { Enunciado oracional } \\
\text { (signo: dos puntos) }\end{array}$ & $\begin{array}{l}\text { Explicación, aclaración, resumen, ampliación, } \\
\text { consecuencia o causa de algo anunciado en el } \\
\text { segmento previo. }\end{array}$ \\
\hline & $\begin{array}{l}\text { Sintagma } \\
\text { (signo: coma) }\end{array}$ & $\begin{array}{l}\text { Elemento adjunto, añadido; inciso; elemento explicativo } \\
\text { o circunstancial; elemento individual en una serie. }\end{array}$ \\
\hline
\end{tabular}


La primera investigación profunda, y realizada con un aparato lingüístico claro y bien fundado, acerca de la expresión escrita de los estudiantes en Costa Rica toca apenas de paso el asunto de la puntuación. Nos referimos a Rodino y Ross (1985), quienes proponen un análisis detallado para aspectos morfosintácticos y léxicos, pero no así para la ortografía, la puntuación y la caligrafía, que son tratadas en conjunto en dos páginas. Para estos autores, los problemas de puntuación responden al mismo problema de fondo que presentan los demás elementos estudiados: "la transferencia de pautas orales a la escritura".

Después de aclarar que el poco y mal uso de los signos de puntuación pertinentes por parte de los sujetos de su estudio resulta evidente, Rodino y Ross afirman que sus textos se muestran confusos y extensos, con una pobre separación y organización de los conceptos; todo esto debido a que el redactor plasma por escrito el "flujo de la producción lingüística oral" (Rodino y Ross, 1985:37). A esto se aúna el problema de que los sujetos no saben discriminar cómo ni cuándo utilizar los signos, por lo que terminan haciéndolo de forma arbitraria.

En el mismo año, aparece el diagnóstico sobre el rendimiento académico de estudiantes de primaria y secundaria. En este estudio, Rojas et al (1985) evalúan distintos aspectos relativos a la asignatura académica denominada español en el $20 \%$ de la población estudiantil de todos las instituciones oficiales de Tercer Ciclo y Educación Diversificada (43 colegios). Para las escuelas, se trabajó con 127 de ellas. Los resultados en cuanto a la puntuación son calificados por las autoras como "críticos", pues el mayor porcentaje de los alumnos de cuarto, sexto y sétimo años obtuvo el nivel mínimo en este rubro.

También Rojas y Umaña (1986ab) tratan dentro de la misma sección la ortografía y los signos de puntuación al exponer los resultados de su investigación sobre el uso del registro escrito por parte de alumnos de undécimo año. Mediante una metodología estadística y un sistema de puntaje con escalas de nivel de propiedad en el manejo de determinados ítemes, estos autores afirman que la mayoría de la población estudiada por ellos se ubica en los cero puntos, es decir, un empleo inadecuado de los signos: únicamente un 2,5\% usó apropiadamente la coma, mientras que más de las tres cuartas partes no utilizaron los dos puntos, los signos de admiración y de interrogación, el guión, la raya, el paréntesis, los puntos suspensivos y las comillas. Por otra parte, los signos empleados (la coma, el punto y aparte y la sangría) se utilizaron erróneamente en muchos casos. Por ello, los autores concluyen: "No hay un conocimiento aceptable de los signos de puntuación. Los que se usan no se dominan (coma, punto y coma, punto y seguido, y punto y aparte) y los demás, que constituyen la mayoría, no aparecen. En consecuencia, los signos de puntuación empleados son los básicos, con el agravante de que están mal usados" (Rojas y Umaña, 1986:71). Los autores afirman que este subempleo "manifiesta desconocimiento de las articulaciones de los elementos que componen el enunciado" (Rojas y Umaña 1986:106). Sin embargo, y a pesar de que pareciera que estos autores reconocen el papel textual de la puntuación, también se deja entrever un criterio "oralista" en su concepción cuando aseveran que "los signos que se actualizan no representan adecuadamente las pausas del registro oral" (íbidem:111).

A una conclusión similar llegó Jiménez Montero (1987), quien únicamente apunta que el descuido en cuanto al uso de los signos de puntuación es notable en las redacciones estudiadas por él (correspondientes al $26 \%$ de la población de primer año de universidad de la Sede del Atlántico de la Universidad de Costa Rica). Para este autor, no existe el más elemental conocimiento de este tema.

Chavarría (1988), en su investigación basada en 380 redacciones de colegiales, 
califica la falta de uso del punto final como marcador de final de párrafo como una omisión "epidémica". Este autor registra más de 650 omisiones de este signo en las redacciones cortas, aunque esta ausencia es mínima en los dictados, lo que él achaca a la posible señal prosódica (tono relativamente bajo) en este caso. También menciona de paso que se da una casi total ausencia del punto y coma, los dos puntos y la coma, y que el uso de estos signos depende mayoritariamente de convenciones meramente ortográficas. Chavarría se queda en estas lacónicas anotaciones sin dedicar más comentarios con respecto al uso de los signos de puntuación en los escritos estudiados por él.

En resumen, a pesar de que los problemas de puntuación en la comunidad costarricense han sido estudiados con anterioridad a este trabajo, creemos que el aparato conceptual con el que se ha abordado el asunto carecía de la especificidad necesaria para estudiar la puntuación. Además, la presentación de los resultados es tan escueta que difícilmente se puede llegar a una comprensión cabal del fenómeno.

\section{Análisis del uso de los signos de puntuación y su relación con las unidades textuales en redacciones de estudiantes universitarios costarricenses}

En este trabajo se analizó un corpus de 60 redacciones de estudiantes de primer ingreso a la Universidad de Costa Rica: 30 provenientes de colegios públicos y otros 30 de colegios privados. Este corpus es el material de base para una investigación de mayor envergadura que estamos llevando a cabo desde un enfoque discursivo.

En vista de que el interés de este trabajo era evaluar el uso de los signos de puntuación como marcas de segmentación de los textos; es decir, su uso real por parte de los estudiantes universitarios en textos totalmente elaborados por ellos, no se consideró pertinente someterlos a ninguna prueba de puntuación de textos sin segmentar o de oraciones con usos concretos. Las redacciones estudiadas debían referirse a algún problema actual en Costa Rica y sus posibles soluciones; además, se les dio a los sujetos la indicación de que los textos debían considerarse como una tarea con valor para la nota final de un curso universitario y que estos serían leídos por alguno de sus profesores. Con lo anterior, se pretendía hacer la indicación implícita de que se trataba de un texto de índole académica, para así evitar cualquier empleo de un estilo literario. De esta forma, podemos examinar la puntuación de las redacciones en cuestión como la propia de textos de estilo formal académico (como los que utiliza Figueras 2001, para elaborar su propuesta).

En el Cuadro 4, se puede observar el conteo total de todos los signos de primer régimen; esto es, las marcas de segmentación de unidades textuales y de indicación de sentido. Para los diversos usos específicos que se analizaron en algunos signos, se siguió la propuesta de Figueras (2001). Luego se procedió a evaluar si el signo estaba bien o mal utilizado en función de los criterios mencionados (segmentación y guía interpretativa). A este respecto es necesario aclarar que un estudio del uso de los signos de puntuación requiere cierta flexibilidad si se asume una postura pragmático-discursiva, en el entendido de que se trata de evaluar la utilización de tales marcas como elementos de cohesión en textos concretos con parámetros como la eficacia (economía en el empleo de los recursos lingüísticos) y la efectividad (impacto del uso de tales recursos), por sobre un criterio de tipo normativo. Así, por ejemplo, podría ser que un estudiante no haya marcado un determinado inciso por medio de comas, pero que, por la naturaleza del inciso y por su ubicación y relación con el enunciado donde aparece inserto, ello no implique un perjuicio para el sentido ni la buena segmentación del discurso; en tales casos, por lo tanto, no se procedió a contabilizarlo como un mal empleo. 
Como se puede observar en el Cuadro 4, los signos más empleados son el punto (punto y aparte y punto y seguido) y la coma; esta última en un porcentaje a todas luces mucho mayor. Estos resultados no resultan nada sorprendentes si se recuerda lo ya señalado por Rojas y Umaña (1986) en relación con textos escritos por estudiantes de undécimo año: se utilizan únicamente los signos más básicos.

En principio, tanto la interpretación de Rojas y Umaña (1986) como nuestros datos estarían en consonancia con la posición de Luna (Cassany, 1995) de distinguir seis grados de complejidad en la puntuación de los textos. Según este autor, cada grado de complejiidad incluye los signos contemplados en los grados inferiores a él, por lo cual se avanza en un contínuum de menor a mayor complejidad conforme se encuentren en los textos las siguientes marcas: 1. punto y seguido; 2. punto y aparte y coma; 3. punto y coma; 4. dos puntos; 5. puntos suspensivos; 6. guiones, paréntesis, comillas y recursos para resaltar. De este modo, por ejemplo, solamente diecinueve estudiantes emplearon correctamente alguna vez el punto y coma en sus redacciones, lo que revelaría el subempleo de los signos de los grados de complejidad 3 en adelante.
El cuadro nos da una idea más clara, asimismo, de las unidades textuales que representan mayores problemas para los sujetos de nuestro corpus: la cláusula textual y el sintagma. A continuación realizamos un análisis detallado de cada signo, la respectiva unidad que marca, y los usos específicos que se observaron.

\subsection{El uso del punto}

En esta sección, examinaremos el uso del punto en sus tres funciones segmentadoras: punto final, punto y aparte, y punto y seguido.

Como se aprecia en el Cuadro 4, en el $78 \%$ de los casos, los estudiantes emplearon bien el punto final. Los principales problemas en su uso son de dos tipos: su ausencia (6 casos), lo que implica que falta la marca formal que indica que la unidad 'texto' llegó a su fin; y su presencia (7 casos) en textos que difícilmente se podrían considerar como concluidos, ya sea porque el final se produce de forma abrupta, porque las unidades informativas están desarticuladas, o porque no se cierra efectivamente la unidad 'texto' (no hay conclusión).

Como se ve, el segundo criterio para considerar como inapropiado el uso del punto final no está basado únicamente en

Cuadro 4

Totales en el uso de los signos de puntuación para cada unidad textual

\begin{tabular}{|l|c|c|c|}
\hline SIGNO Y UNIDAD TEXTUAL & Total & Bien empleado & Mal empleado \\
\hline Punto final-TEXTO & $60 / 2,5 \%$ & $47 / 78 \%$ & $13 / 22 \%$ \\
\hline Punto y aparte- PÁRRAFO & $340 / 14,5 \%$ & $264 / 78 \%$ & $76 / 22 \%$ \\
\hline Punto y seguido-ENUNCIADO TEXTUAL & $385 / 16,5 \%$ & $316 / 82 \%$ & $69 / 18 \%$ \\
\hline Punto y coma-CLÁUSULA TEXTUAL & $57 / 2,5 \%$ & $32 / 56 \%$ & $25 / 44 \%$ \\
\hline Dos puntos-ENUNCIADO ORACIONAL & $46 / 2 \%$ & $34 / 74 \%$ & $12 / 26 \%$ \\
\hline Coma-SINTAGMA & $1469 / 62 \%$ & $1000 / 68 \%$ & $469 / 32 \%$ \\
\hline TOTAL & $2357 / 100 \%$ & $1693 / 72 \%$ & $664 / 28 \%$ \\
\hline
\end{tabular}


la observación simplista de su presencia o ausencia, sino en la consideración de la calidad de las redacciones de los estudiantes en cuanto textos que deben cumplir con ciertas características esenciales para ser aceptados como tales: desarrollo de un tema, cohesión de sus distintas unidades, coherencia informativa, estructuración.

En cuanto al punto y aparte, su uso determina el manejo adecuado de la unidad conocida como 'párrafo'. Por ello mismo, el uso adecuado de este signo es evidencia de un manejo apropiado de la distribución de la información en los textos, lo cual repercute en la facilidad, por parte del lector, para seguir la lectura y para procesar el discurso. El punto y aparte indica que se concluyó una sección informativa en torno a una idea que guarda relación con el texto global, porque desarrolla un subtema del texto o porque se segmenta el tema general en ideas particulares. El párrafo debe mostrar coherencia interna (los enunciados que lo componen giran en torno a la misma idea tópica) y coherencia externa (el subtema tratado debe estar relacionado con el tema del texto) (Garachana y Montolío, 2000).

Precisamente, el principal problema en cuanto al uso de este signo en realidad guarda relación con el uso que hacen los sujetos del párrafo como mecanismo de distribución de la información; es decir, se nota una deficiencia en el manejo de esta unidad textual por parte de los sujetos en estudio, según se constata en el uso erróneo de este signo en un $22 \%$ de los casos contabilizados. Esta impropiedad se evidencia en párrafos con un sentido vago y confuso, párrafos que no desarrollan ninguna idea en concreto (y que no son unidades de delimitación, como los de introducción), párrafos con varias ideas principales (con sobrecarga semántica), párrafos que se dividen en dos cuando no se justifica, párrafos con ideas que no se relacionan con el texto (párrafos "ociosos"). El siguiente fragmento servirá para ejemplificar un caso de un párrafo sobrecargado de información sin desarrollar (el primero) seguido por uno totalmente innecesario y desligado del texto (el tema es la contaminación ambiental $)^{9}$ :

"Como posibles soluciones, solo veo la necesidad que tiene este país conocido en todo el mundo como "Paraíso ecológio" de cambiar su mentalidad. Si la gente no entiende con publicidad ecologista, la única manera de hacer entender será emitiendo multas, osea hacer una política ambientalista actual, los tiempos cambian y las políticas deben cambiar con él, además implantar en las escuelas del país un sistema educativo que mencione la importancia de la naturaleza y como la estamos afectando. Se debe poner orden a las municipalidades y ver si el presupuesto dedicado a la recolección de basura esta siendo aplicado en su verdadero propósito. Por los ríos y mantos acuíferos contaminados la única solución es buscar ayuda de países que tengan la tecnología necesaria para el rescate de estos lugares, por ejemplo Holanda.

Claramente Costa Rica nos es único país en el mundo con esta clase de problemas, cientos de países han sufrido catástrofes con derrames de petróleo, radiación, etc.

Soy consiente de que las soluciones que propongo requieren una gran cantidad de dinero para llevarse a cabo, pero si se desea conservar esta fama de Paraíso Ecológico se deben "tomar cartas en el asunto" lo más rápido posible.” (A-25).

Por último, tendríamos el punto y seguido, concebido por Figueras (2001) como un elemento de tránsito entre el nivel macroestructural y el nivel microestructural y especializado en señalar que el escritor concluye un aspecto temático específico acerca del subtema tratado en el párrafo. Según Figueras, para poder emplear adecuadamente este signo se requiere tomar en cuenta dos criterios: uno de índole semántica (detalle temático) y uno de naturaleza sintáctica (segmentos no enlazados con otros por medio de conectores de coordinación ni de elementos de subordinación). Esta misma autora señala que precisar los límites semánticos de la unidad 'enunciado textual' es difícil, pues depende en gran medida de cómo el escritor desea distribuir la información en el párrafo y del tipo de texto. Sin embargo, dentro de este ámbito de libertad en el que el autor puede utilizar la puntuación con los fines pragmático-semánticos 
que tenga a bien, la conjunción de los criterios mencionados sirve de base para evaluar los problemas en los que incurren los estudiantes.

Según nuestro análisis, el uso adecuado de este signo supera con creces su empleo inapropiado: solamente registramos un $18 \%$ de usos incorrectos. Los principales problemas se deben a una infrasegmentación (uso del punto y seguido cuando lo que corresponde es el punto y aparte), a sustitución (especialmente por la coma), a su uso en lugar de otro signo más apropiado (como los dos puntos). En los siguientes ejemplos, el primer párrafo muestra un punto y seguido que corta la relación semánticosintáctica y que, por ende, dificulta enormemente la comprensión del fragmento (tema: la xenofobia); el segundo párrafo, por su parte, muestra problemas de segmentación (uso de comas en lugar de puntos y seguidos, y falta de coherencia interna por la yuxtaposición de ideas sin relación unas con otras) (tema: embarazo de adolescentes):

\footnotetext{
"Subsiguiente estos valores serán reforzados con programas de comunicación de masas. A la población en general; suena soñador e imposible pero el objetivo primordial es cambiar la mentalidad de las personas con el tiempo, pues, si fue posible inculcarles ese odio, será realizable desarraigarlo, será viable privarles el derecho a odiar a sus semejantes por diferencias, consumando que debajo de esa piel, muy por encima de esas características étnicas todos somos humanos, seres homogéneos con un mismo origen y fin”. (A-29)
}

"Estos terribles acontecimientos ocurridos con una regularidad asombrosa deben de ser acabados, el Patronato Nacional de la Infancia (PANI) debe, (como es su deber) hacer campañas, enviar personas especializadas en temas como sexualidad, prevención de embarazos a ofrecer charlas en escuelas, realizar programas en los medios de comunicación entre otros, para que la mayoría de los niños conozcan sobre los mismos y así evitar que sigan sucediendo estos hechos. Es necesario que se castigue duramente a las personas que sin ninguna justificación pueden cometer semejantes atrocidades. En la mayoría de los casos los hechos criminales son realizados por familiares de la víctima, esto demuestra que el conocimiento y la práctica de los valores en la familia son nulos". (A-10).

\subsection{El uso del punto y coma}

El punto y coma funciona en el interior del enunciado textual y se distingue del punto y seguido en función del parámetro de proximidad semántica. Con el punto y coma, se muestra una relación temática más estrecha entre dos segmentos informativos que si se emplea el punto y seguido, aunque esos dos segmentos mantengan independencia sintáctica. Los tres usos básicos de este signo contemplan la unión de unidades yuxtapuestas; la separación de estructuras consecutivas, concesivas, etc. (especialmente cuando se echa mano de conectores que indican tales relaciones lógicas, como 'por lo tanto' o 'sin embargo'); y la separación de enumeraciones complejas (cuando existe una puntuación interna dentro de los elementos enumerados, como sucede en este mismo párrafo). El Cuadro 5 resume los datos de nuestro corpus a este respecto.

Como se observa, este signo se emplea poco en las redacciones analizadas, lo cual quizás revela un grado de complejidad bajo en la estructuración de los textos, como se anotó al inicio de este apartado. Aunado a su poco uso, se nota un manejo inadecuado como estrategia para mostrar relación semántica entre unidades yuxtapuestas de un mismo enunciado: $44 \%$ de usos impropios por parte de los estudiantes.

El empleo de este signo para separar unidades de relación lógica (consecuencias, causas, etc.) apenas si aparece en nuestro corpus. La situación de las enumeraciones complejas es aún más precaria (solamente un caso -y mal empleado- en las 60 redacciones). Podría pensarse que, en este aspecto en especial, se nota la poca complejiidad informativa y estructural de los textos examinados. El siguiente ejemplo muestra un caso de empleo adecuado de este signo (tema: pérdida de identidad):

"Por otra parte, Costa Rica, al ser un país de poco poder y con una débil figura de identidad, se ve especialmente perjudicada con el fenómeno de la globalización 
Cuadro 5

Usos del punto y coma

\begin{tabular}{|l|c|c|c|}
\hline Uso del punto y coma & Bien empleado & Mal empleado & Total \\
\hline Unidades yuxtapuestas & $27 / 53 \%$ & $24 / 47 \%$ & $51 / 89 \%$ \\
\hline $\begin{array}{l}\text { Estructuras causales, consecutivas, } \\
\text { condicionales, etc. }\end{array}$ & $5 / 100 \%$ & 0 & $5 / 9 \%$ \\
\hline Enumeraciones complejas & 0 & $1 / 100 \%$ & $1 / 2 \%$ \\
\hline Total & $32 / 56 \%$ & $25 / 44 \%$ & $57 / 100 \%$ \\
\hline
\end{tabular}

por dos razones primordiales: primero la actitud imitativa a la que tienden la mayoría de los costarricenses, quienes adoptan las culturas extranjeras más fuertes y las toman como propias; esta situación provoca que los nacionales se olviden de sus raíces y su verdadera cultura (...)" (A-6)

En el siguiente ejemplo, se puede ver el uso apropiado de este signo ante un conector contraargumentativo (adversativo), justificado por la forma en que se presenta la información en el párrafo (tema: fiascos viales):

"Además, el dinero no debe manejarse a la ligera. Es necesario plantear cuentas claras, definir el tiempo de construcción en base al costo y por qué no, contar con un fondo de emergencias; pero no cualquier tipo de emergencias, sino aquellas que de verdad ameriten a las instituciones que anteriormente anoté" (B-3).

Los siguientes dos fragmentos ilustran los problemas en el uso de este signo. En el primero, estamos ante un texto casi sin puntuar, en el que las relaciones semántico-sintácticas están débilmente indicadas y en el que se nota la falta de puntos y coma (entre otros signos, principalmente el punto y seguido) para segmentar las unidades de información (tema: violencia doméstica). En el segundo, se utiliza el punto y coma en una enumeración compleja. El problema reside en que se separan, por medio de punto y coma, dos series de elementos, como si constituyeran dos grupos cuyas unidades son homogéneas entre sí y distintas de las de la otra serie; sin embargo, no se trata de dos grupos distintos, sino de un solo conjunto en el que se halla un elemento que lleva un inciso. En este sentido, lo apropiado hubiera sido separar cada elemento con un punto y coma o insertar el inciso dentro de paréntesis (tema: la falta de planificación):

“(...) No obstante en algunas ocasiones llegan a involucrar a segundas personas como son los hijos, este problema se centra en menores de edad, cuando ellos ven a sus padres peleando empiezan a pensar que es su culpa y esto puede dañar seriamente su crecimiento, su rendimiento escolar, se pueden a empezar a hacerse agresivos y lo que es peor es que esto se puede volver hereditario y causar un trastorno que sería desastrozo (...)" (B-5).

"Así como nosotros, nuestros hijos tienen el derecho de disfrutar de una democracia, de una vida tranquila; sin guerras ni hambre, de esos majestuosos bosques que le dan vida a nuestra tierra, de todo lo lindo que los costarricenses poseemos. Y es nuestro deber darles ese orgullo de ser ticos. Ese orgullo que gracias a nuestros abuelos llegamos a sentir" (B-27).

\subsection{El uso de los dos puntos}

Los dos puntos indican que el segmento informativo antecedido por estos constituye una continuación semántica de la porción que los precede; en otras palabras, anuncian que a continuación sigue un fragmento que complementa, amplía, explica o sintetiza la información del segmento previo. Por lo general, marcan que la información que los sigue es una consecuencia, una causa, una reformulación, un 
resumen, una aclaración o una conclusión de lo dicho anteriormente. El Cuadro 6 recoge los principales usos de este signo y los casos de su empleo en nuestro corpus.

Se nota que los usos manejados por los estudiantes son solamente los correspondientes a introducción de enumeraciones, de conclusiones y de anuncio de una porción informativa. No apareció ni un solo ejemplo de introducción de causas o consecuencias, y muy pocos de ejemplificaciones y citas textuales. Llama la atención que, pesa a su subutilización, es igual el número de veces que se empleó apropiada e inapropiadamente en las citas textuales, y más las veces en las que se utilizó mal en las ejemplificaciones. El uso de este signo para anunciar una causa o un resultado está totalmente ausente en el corpus analizado.

Un ejemplo de ausencia de este signo en un entorno en que se requiere casi obligatoriamente lo tenemos a continuación (tema: la política). Sin este signo, la interpretación del fragmento se torna dificultosa:

"Promesas, sueños, cielo y tierra ofrecidos pero al final no son más que eso promesas que se olvidaran cuando se ha logrado llegar a donde se quería, ahí ya todo es diferente, se olvidan de las tantas personas que por ellos votaron, sabiendo que es por ellos que lograron llegar ahí" (B-2).

\subsection{Usos de la coma}

La coma es el signo con la mayor diversidad de usos, el más empleado en las redacciones examinadas y el que más problemas presenta. En general, la coma separa sintagmas, elementos periféricos o aclaratorios, constituyentes de un mismo enunciado, pero no elementos fuertemente ligados en la estructura sintáctica. Por ello mismo, se considera un uso inapropiado separar con coma el sujeto y el predicado de una misma cláusula. Es, además, el signo que más injustamente se asocia con la indicación de pausas del registro oral, lo cual sin duda conlleva a su sobreutilización. El Cuadro 7 muestra los resultados de nuestro análisis.

En primer lugar, se aprecia cuál es el uso de la coma más empleado por los sujetos: la segmentación en sintagmas. Lo siguen las enumeraciones y los conectores. El uso de la coma como marcador de elisiones sintácticas está prácticamente ausente.

En lo concerniente a la segmentación de sintagmas, el uso inapropiado de este signo reporta un porcentaje considerable.

Cuadro 6

Usos de los dos puntos

\begin{tabular}{|l|c|c|c|}
\hline Uso de los dos puntos & Bien empleado & Mal empleado & Total \\
\hline Enumeraciones & $18 / 95 \%$ & $1 / 5 \%$ & $19 / 41 \%$ \\
\hline Citas textuales & $3 / 50 \%$ & $3 / 50 \%$ & $6 / 13 \%$ \\
\hline Ejemplificaciones & $1 / 20 \%$ & $4 / 80 \%$ & $5 / 11 \%$ \\
\hline Introducción de causas & 0 & 0 & 0 \\
\hline Introducción de efectos & 0 & 0 & 0 \\
\hline Introd. de conclusiones, resumen, anuncio & $12 / 75 \%$ & $4 / 25 \%$ & $16 / 35 \%$ \\
\hline Total & $34 / 74 \%$ & $12 / 26 \%$ & $46 / 100 \%$ \\
\hline
\end{tabular}


Cuadro 7

Usos de la coma

\begin{tabular}{|l|c|c|c|}
\hline Uso de la coma & Bien empleado & Mal empleado & Total \\
\hline Incisos & $97 / 44 \%$ & $124 / 56 \%$ & $221 / 15 \%$ \\
\hline Cambios de orden & $48 / 79 \%$ & $13 / 21 \%$ & $61 / 4 \%$ \\
\hline Elementos supraoracionales & $7 / 78 \%$ & $2 / 22 \%$ & $9 / 1 \%$ \\
\hline Enumeraciones & $240 / 95 \%$ & $12 / 5 \%$ & $252 / 17 \%$ \\
\hline Conectores & $223 / 73 \%$ & $84 / 27 \%$ & $307 / 21 \%$ \\
\hline Elisiones & $1 / 100 \%$ & & $1 /(0,06 \%)$ \\
\hline Sintagmas & $384 / 62 \%$ & $234 / 38 \%$ & $618 / 42 \%$ \\
\hline Total & $1000 / 68 \%$ & $469 / 32 \%$ & $1469 / 100 \%$ \\
\hline
\end{tabular}

La mayoría de los errores consisten en la separación del sujeto y el predicado o del verbo y alguno de sus complementos directos, indirectos o complementos verbales (38 casos). También es común el uso de la coma en sustitución de muchos otros signos (puntos y seguido, puntos y coma, dos puntos, paréntesis y guiones: 118 casos). Algunos de estos problemas se ilustran a continuación.

Separación del sujeto y el predicado:

"Es de suma importancia, contar con individuos que conozcan los proyectos" (B-3).

“(...) realmente lo que se les esta enseñando, les servirá en el desarrollo de sus Facultades en un futuro" (A-16).

"Otro factor por el cual muchas adolescentes quedan embarazadas, son los abusos sexuales o violaciones" (B-19).

Separación del verbo y uno de sus complementos fuertes:

"Estos padres de familia aluden a la realidad nacional, de que la matemática es la materia que presenta los resultados más bajos a nivel de noveno año y bachillerato" (A-17).
En el siguiente ejemplo, se nota su uso en reemplazo de otros signos más adecuados, que muestren realmente una jerarquización informativa. El párrafo da la sensación de ser una yuxtaposición de ideas inconexas:

"En los casos en que las jóvenes son abusadas sexualmente sus padres se preocupan por buscar al culpable y hacerlo pagar por lo ocurrido. La propuesta que se presento hace poco de que a una niña se le practicara un aborto fue un tema de mucha discusión a nivel nacional, su embarazo era producto de una violación, al supuesto culpable no se le pudo probar nada, sus padres querían que se le hiciera el aborto pero los médicos y las autoridades costarricenses no lo aprobaron" (B-18).

No obstante, la mayor cantidad de usos inadecuados se halla en la coma como marcador de incisos textuales, lo cual revela que es este el empleo que mayores dificultades presenta para los estudiantes (56\% de los casos de incisos fueron mal puntuados). Este problema en el uso de la coma y en la segmentación de los textos revela, en realidad, una dificultad para determinar cuáles son los elementos que se insertan en la línea informativa del 
sintagma o de la cláusula. Este problema, por lo general, se muestra en una puntuación deficiente que coloca una coma antes o después del inciso, pero no las dos que corresponden para marcar su carácter de elemento explicativo parentético, como se puede observar en los siguientes ejemplos, en los que la dificultad para interpretar o procesar la información varía de acuerdo con el entorno sintáctico:

"Como veran [,] todos los costarricenses estamos en riesgo tan solo acudiendo a aquellos lugares" (B-8).

"Se puede destacar que [,] si cada persona se arma de valor y se dispone a preocuparse más por su propia seguridad, tomando cursos de defensa personal, por ejemplo, no sólo va a sentirse más segura y tranquila que antes, sino que también aprenderá como autoestimarse" (B-11).

"En fin, si trabajamos todos en conjunto y, de corazón [,] deseamos una mejora, podremos lograr que nuestras voces sean escuchadas" (B-13).

“(...) así que no me puedo imaginar si quiera que pasa por la cabeza de esta niñita que [,] aunque creo que entiende que le pasa [,] no puede comprender totalmente las dimensiones de su problema” (A-18).

\subsection{Los signos de segundo régimen: Introducción de un segundo discurso}

Entre los signos que no marcan una segmentación primaria del texto, encontramos tres elementos que se emplean en español para insertar un segundo discurso; es decir, segmentos que aportan datos suplementarios que ayudan a comprender la información o que la detallan, o fragmentos tomados literalmente de otro autor. En este sentido, los paréntesis y guiones largos actúan como marcadores de incisos; mientras que las comillas están especializadas para hacer sobresalir citas textuales. Para los efectos de este trabajo, se van a omitir los datos correspondientes a las comillas, por cuanto el uso que hicieron de ellas los estudiantes no guarda relación con la introducción de un segundo discurso, sino con la actitud del autor (dotar a una palabra de un sentido irónico, llamar la atención sobre un término). Los empleos de los otros dos signos en las redacciones de nuestro corpus se muestran en el Cuadro 8.

Lo primero que salta a la vista es la subutilización de los guiones largos para marcar incisos. Tampoco los paréntesis se emplearon como un recurso idiomático a este respecto, pues, de los pocos usos encontrados, la mayoría corresponde a aclaraciones de términos y no a puntualización o inclusión de información secundaria. La explicación de este infraempleo lo hallamos nuevamente en la sobreutilización de las comas.

Tanto las comas como los paréntesis y los guiones largos sirven para marcar incisos textuales. La diferencia estriba en que las comas segmentan incisos primarios altamente vinculados a la estructura semántica e integrados en la estructura sintáctica de los segmentos informativos en los que se insertan. Por su parte, los guiones y paréntesis indican que los incisos están menos integrados, menos vinculados, son más secundarios. La distinción entre ambos signos está relacionada con la actitud del autor: los guiones se emplean comúnmente para introducir comentarios personales del que escribe.

Cuadro 8

Signos de segundo régimen: Introducción de un segundo discurso

\begin{tabular}{|l|c|c|c|}
\hline Signo de puntuación & Bien utilizado & Mal utilizado & Total \\
\hline Guiones & $1 / 17 \%$ & $5 / 83 \%$ & 6 \\
\hline Paréntesis & $19 / 83 \%$ & $4 / 17 \%$ & 23 \\
\hline
\end{tabular}


En nuestro corpus, se nota cómo los autores recurrieron a otros signos de puntuación (especialmente la coma) para marcar incisos de todo tipo, independientemente del grado de integración semántico-sintáctica de estos, lo cual produce -en no pocos casos- una ruptura en la línea informativa de los textos que dificulta su procesamiento, como en los siguientes ejemplos, en los que el uso de guiones largos y paréntesis hubiera facilitado la jerarquización informativa:

"Los periodistas con comentarios incompletos pienso; atacaban la paz" (B-21).

"Queremos que se impartan doscientos días lectivos cuando no estamos preparados para alimentar a los jóvenes porque se robaron los fondos asignados a este fin; cuando no hay suficientes aulas mucho menos pupitres y cada vez el costo del transporte sube de una manera exorbitante" (A-16)

"Cuando se trata de construir o arreglar una carretera en Costa Rica es un proceso muy largo y difícil, en el sentido de que el gobierno dura mucho tiempo aceptando el plan de construcción, me refiero a unos varios años, y cuando por fin lo acepta el plan ya esta desactualizado con la sociedad actual, dado que los automoviles en circulación aumentan considerablemente cada año" (A-23).

El hecho de que no es muy claro para estos sujetos el valor textual de estos signos de puntuación se hace más que patente en el siguiente caso, en el cual el autor encierra un comentario personal entre paréntesis - pues reconoce que se trata de un inciso-, pero coloca antes una coma. Evidentemente, se trata de una mezcla de recursos:

"Estos terribles acontecimientos ocurridos con una regularidad asombrosa deben de ser acabados, el Patronato Nacional de la Infancia (PANI) debe, (como es su deber) hacer campañas, enviar personas especializadas en temas como sexualidad, prevención de embarazos a ofrecer charlas en escuelas, realizar programas en los medios de comunicación entre otros, para que la mayoría de los niños conozcan sobre los mismos y así evitar que sigan sucediendo estos hechos.

Asimismo, es patente el desconocimiento del valor textual de estos signos en el ejemplo que sigue, en el cual se coloca entre paréntesis información que debe ir integrada al texto:

"Ellos se enfrentan, primero, a problemas como la venta de drogas ilegales entre estudiantes, así como, problemas académicos tales como deserción y abandono escolar que crea patrones delictivos en los adolescentes, en pocas palabras, (chapulines y pandillas)" (B-8).

A pesar de los problemas en el manejo de estos recursos de cohesión que hemos señalado, valga aclarar que también encontramos casos de una utilización adecuada y eficaz de estos signos:

“(...) Costa Rica -como sucede verdaderamente- puede sacar gran provecho económico” (B-20).

“(...) porque el conocimiento del capital humano (definido por el aporte que la educación hace al crecimiento económico del país) es un factor primordial en la atracción de la inversión extranjera al país” (B-20).

\subsection{Los signos de segundo régimen: Marcadores de modalidad}

Finalmente, existen unos signos que no se utilizan tanto para segmentar en unidades informativas un texto como para mostrar la actitud del autor frente a lo que escribe. No se entrará aquí en disquisiciones teóricas sobre la categoría de modalidad; baste, para los efectos de este trabajo, indicar que los signos de interrogación y de exclamación, junto con los puntos suspensivos, sirven para guiar la interpretación del texto en cuanto a la posición de su escritor con respecto a la información contenida. El Cuadro 9 resume nuestros hallazgos.

Como era de esperar en textos de carácter académico como los examinados aquí, los signos de exclamación aparecen muy poco, al igual que los puntos suspensivos. Los signos de interrogación se utilizan como marcadores de estrategias discursivas para captar la atención del lector con respecto a lo que se va a informar posteriormente (interrogaciones expositivas) 


\section{Cuadro 9}

Signos de segundo régimen: Marcadores de modalidad

\begin{tabular}{|l|c|c|c|}
\hline Signo de puntuación & Bien utilizado & Mal utilizado & Total \\
\hline Pregunta & $33 / 53 \%$ & $29 / 47 \%$ & 62 \\
\hline Exclamación & $4 / 100 \%$ & 0 & 4 \\
\hline Puntos suspensivos & $12 / 100 \%$ & 0 & 12 \\
\hline
\end{tabular}

o para persuadir sin que se aprecie que el autor está imponiendo su posición (preguntas retóricas). Los siguientes fragmentos ejemplifican estos usos:

"¿Tiene usted a algún familiar, amigo o conocido que haya venido para buscar una mejor calidad de vida, libertad de expresión? El porcentaje de inmigrantes en nuestro país aumenta cada día más, causando que la población crezca sin ninguna planificación” (A-20).

"Nuestra sociedad está tambaleante. ¿Cuántas veces hemos escuchado esta frase? Ya sea en discursos impartidos por nuestros políticos, noticias televisivas o radiales, talvez incluso dentro de nuestro mismo hogar. ¿Pero qué significan realmente esas palabras? No tenemos que remontarnos mucho tiempo atrás para darnos cuenta de que nuestro entorno ha ido cambiando y no todo el tiempo para bien.” (B-13).

El principal problema en el uso de este último signo consiste en la ausencia de marcación de las interrogaciones o en el empleo de únicamente el signo de cierre. En los siguientes ejemplos, nótese cómo dificulta la interpretación de los fragmentos la ausencia de los signos de interrogación correspondientes:

"[i]Que derecho existe, cuando por un numero pequeño de personas, se juzga y condena a todo un pueblo, hambriento de justicia, azotado por calamidades de la pobreza como cualquier otro, que tiene su esperanza fijada en nuestra nación[?], gente perteneciente a diversas partes del mundo seleccionan a nuestro país como destino para expandir sus oportunidades y enriquecerse culturalmente" (A-29).

"Otro caso es la dela pequeña que fue embarazada por el mismo hombre que embarazo a su madre o la de la pequeña de doce años que ya es madre de una niña de dos meses y cuyo culpable de haberle cambiado la vida esta libre bajo el hecho deque dijo que se haría cargo de la niña y la bebe. [¿]Como queremos cambiar estas realidades si no damos ni un poco de justicia[?][¿]Será que acaso como leí en un reportaje hace poco la justicia muerto[?]" (B-22).

\section{Conclusiones}

"Vamos a ponernos de acuerdo en algo esencial. Los signos de puntuación no se escriben para indicar las pausas respiratorias. Primero que todo, porque son muy pocos los textos destinados a la lectura en voz alta $y$, segundo, porque la puntuación variaría según el ritmo respiratorio del escritor. Es decir, más comas y más puntos, a mayor dificultad para respirar; menos comas y menos puntos, a mayor capacidad pulmonar. ¡Absurdo!

Pero a mí me dijeron en el colegio...

Lo siento mucho. De verdad.

Y si le sirve de consuelo, a mí también me lo dijeron en el colegio (...)".

Fernando Ávila (2001)

Nuestro análisis puede relacionarse fácilmente con las dos conclusiones principales surgidas de las investigaciones sobre el manejo de los signos de puntuación por parte de los estudiantes costarricenses: hay una subutilización de estos recursos y un mal empleo de los signos que aparecen (Rojas y Umaña, 1986), y se nota una interferencia del registro oral en el registro escrito (Rodino y Ross, 1985). No obstante, creemos haber puntualizado mejor el problema del uso de estos signos y su enseñanza, a partir de una perspectiva que privilegia, sobre todo, el valor discursivo de estas marcas. 
Como vimos, se encuentran problemas, principalmente, en la segmentación de las unidades textuales 'párrafo', 'cláusula textual' y 'sintagma'. En particular, los sujetos de este estudio mostraron poca destreza en la marcación de los incisos, los sintagmas y las unidades yuxtapuestas. En cuanto a la coma, se nota su sobreutilización como recurso de reemplazo de otros signos y su colocación en posiciones inapropiadas, quizás como marca de pausa (lo cual se puede relacionar tanto con la mencionada interferencia del código oral como con la enseñanza tradicional de la puntuación). También se comprueba la subutilización del punto y coma, los dos puntos y el punto y seguido, indicadores todos de madurez en las habilidades de expresión escrita.

Creemos -con Figueras, Cassany y Ávila- que, en gran medida, el problema hunde sus raíces en la concepción del valor de los signos de puntuación y su didáctica. A todas luces, el manejo eficaz y eficiente de estos recursos depende de una madurez textual que posibilite desarrollar estrategias de jerarquización informativa (por ejemplo, para separar los incisos), segmentación semántico-estructural (para distribuir la información en párrafos) y sensibilidad gramatical (para separar enunciados y sintagmas, lo cual no debe confundirse con poder etiquetar estructuras según la taxonomía gramatical tradicional).

Si los signos de puntuación muestran su razón de ser en los textos, no tiene sentido que se privilegie su enseñanza a partir de listas de usos aislados ni a partir de nociones "oralistas" o "respiratorias".

En primer lugar, se debe tratar de ayudar al aprendiz a reconocer la necesidad de segmentar bien los textos para guiar su interpretación; luego, se debe practicar constantemente la puntuación en textos elaborados por él. La puntuación es un recurso del registro escrito y, como tal, está al servicio de la construcción textual. Su manejo eficaz no es un fin en sí mismo, sino un medio para fortalecer la expresión escrita; sin embargo, lo ideal es que se pueda contar con él como una herramienta que facilite la escritura y la lectura, y que no se conciba como el contenido de los cursos de redacción que, por excelencia, provoca aversión y dolor de cabeza.

En suma, enseñar puntuación va parejo con enseñar segmentación de los textos. Al enseñar cómo escribir una redacción, cómo elaborar un párrafo, cómo desarrollar una idea central y varias ideas secundarias, cómo incluir información aclaratoria, etc., debemos enseñar también puntuación y viceversa. No hay ninguna práctica más nociva para la didáctica de la composición que enseñar esta destreza "en pedazos", como recursos y usos aislados e inconexos, y luego exigirles a los estudiantes que sean capaces de articular todos esos datos desligados en la construcción de textos apropiados y de calidad. Valdría la pena rescatar, a este respecto, la concepción de los signos de puntuación en su valor arquitectónico, sugerida por Moisés Vicenzi ya en 1930, o en su función similar a las señales de tránsito de la que habla Quijano (1997).

\section{Notas}

1. Probablemente lo hacen de este modo por seguir el modelo de la Real Academia de la Lengua Española, que en sus publicaciones sobre las normas de ortografía incluye el tratamiento de la puntuación. Véase al respecto la última edición de la Ortografía de la lengua española (1999), en la que se afirma: "La puntuación de los textos escritos, con la que se pretende reproducir la entonación de la lengua oral, constituye un capítulo importante dentro de la ortografía de cualquier idioma" (p. 55).

2. Resulta paradógico que los autores mismos aclaren que el estudio de la puntuación es materia más de la sintaxis que de la ortografía, dado que su escritura no está sujeta a cambios.

3. Sin embargo, debemos hacer notar que este libro es un manual de ejercicios y que no presenta listas de usos para cada signo con sus respetivos ejercicios, sino que plantea un método inductivo: 
el estudiante debe descubrir los usos a partir del análisis de fragmentos de ensayos y novelas.

4. La autora eliminará la sección sobre las mayúsculas en su libro La puntuación en el español actual (2002).

5. Los métodos analítico y sintético corresponderían a lo que hoy conocemos como método deductivo y método inductivo, respectivamente.

6. En esencia, se trata del mismo método que propone Figueras (2001), con excepción de que ya no se trabaja a partir de textos literarios, sino académicos.

7. Esta tendencia se debe, muy probablemente, al hecho de seguir las afirmaciones de la Real Academia. Precisamente, según esta institución, "el punto (.) señala la pausa que se da al final de un enunciado" (p. 56), "la coma (,) indica una pausa breve que se produce dentro del enunciado" (p. 58) y "el punto y coma (;) indica una pausa superior a la marcada por la coma e inferior a la señalada por el punto" (p. 66) (ver Real Academia. 1999).

8. "La cohesión constituye una de las más importantes manifestaciones de la coherencia, identificable a partir de elementos lingüísticos visibles y materiales. Se da en el orden interior del texto y funciona como un conjunto de enlaces intratextuales para establecer las relaciones semánticas que precisa un texto para constituirse como unidad de significación" (Calsamiglia y Tusón, 2002: 230). La cohesión es una de las llamadas condiciones de textualidad que sirve para determinar qué constituye un texto (entendido este como evento comunicativo), según la perspectiva de la lingüística del texto (de Beaugrande y Dressler. 1997).

9. Los ejemplos se transcriben tal y como fueron escritos en todos sus aspectos: ortografía, puntuación, gramática, etc. Los códigos que siguen entre paréntesis a los ejemplos sirven como claves para identificar la procedencia del texto: A indica colegio privado y $\mathrm{B}$, público.

\section{Referencias bibliográficas}

Ávila, Fernando. Dónde va la coma. Bogotá: Grupo Editorial Norma. 2001.

Bolaños Calvo, Bolívar. Comunicación Escrita. San José: EUNED. 2001.
Brenes Solano, Rosaura. ¿Cómo comunicarse en forma escrita? San José: EUNED. 1990.

Bustos Arratia, Myriam. Elaboración de esquemas, redacción de notas verbales, presentaciones y recomendaciones. San José: EUNED. 1983.

Bustos Arratia, Myriam. La puntuación al alcance de todos. San José: EUNED. 1994.

Calsamiglia Blancafort, Helena y Amparo Tusón Valls. Las cosas del decir. Manual de análisis del discurso. Barcelona: Ariel. 2002.

Cassany, Daniel. La cocina de la escritura. Barcelona: Anagrama. 1995.

Chavarría, Óscar. La ortografía en perspectiva. Cartago: Ed. Tecnológica de Costa Rica. 1988.

De Beaugrande, Robert-Alain y Wolfgag Ulrich Dressler. Introducción a la lingüistica del texto. Barcelona: Ariel. 1997.

Durán Campos, Vianney y Claudio Jiménez Montero. Un breve estudio de la oración, el párrafo y la puntuación. San Ramón: Taller de Publicaciones de la Sede de Occidente. 1989.

Figueras, Carolina. Pragmática de la puntuación. Barcelona: Octaedro. 2001.

Garachana, Mar y Estrella Montolío. "De la oración al párrafo. Del párrafo al texto.” En: Montolío (coord.). 2000.

Garita, Flor y Maritza Quesada. Prácticas de ortografía. San José: Editorial Nueva Década. 1988.

González Picado, Jézer. El uso de los signos de puntuación en la lengua española. 
San José: Cátedra de Castellano, Universidad de Costa Rica. 1970.

González Picado, Jézer. La comunicación escrita. Curso fundamental de redacción. San José: Alma Máter. 1986.

Jiménez Montero, Claudio. Un aporte al mejoramiento de la expresión escrita. Tesis de Licenciatura en Filología Española, Universidad de Costa Rica. 1987.

Montolío, Estrella (coord.). Manual práctico de escritura académica. Barcelona: Ariel. 2000.

Müller Delgado, Marta Virginia. Puntuación y uso de las mayúsculas en el español actual. San José: M.V. Müller D. 1996.

Müller Delgado, Marta Virginia. La puntuación en el español actual. San José: Editorial Amerrique. 2002.

Pacheco Salazar, Viria y María Angelina Álvarez Flores. Comunicación oral y escrita. San José: G.Q. Impresos. 2000.

Quijano López, Luis Fernando. Español: el cotidiano quehacer del idioma. San José: Editorial Universidad Estatal a Distancia. 1997.

Quilis, Antonio y César Hernández. Lingüistica española aplicada a la terapia del lenguaje. Madrid: Gredos. 1990.

Pazos J., Éthel. El Abecé de la puntuación. San José: Euroamericana de Ediciones. 1990.

Real Academia Española. Ortografía de la lengua española. Madrid: Espasa Calpe. 1999.
Rodino, Ana María y Ronald Ross. Problemas de Expresión Escrita del Estudiante Universitario Costarricense. Un estudio de lingüística aplicada. San José: EUNED. 1985.

Rojas Porras, Marta, Nidia García Lizano y Damaris Fajardo Salas. Diagnóstico evaluativo de la enseñanza del español en la Educación General Básica y Educación Diversificada: rendimiento académico. San José: Instituto de Investigación para el Mejoramiento de la Educación Costarricense. 1985.

Rojas Porras, Marta y Róger Umaña Chacón. Actualización escrita de la variable estándar de la lengua y de los códigos de habla: aportación para una descripción crítica. Tesis de Licenciatura en Filología Española, Universidad de Costa Rica. 1986a.

Rojas Porras, Marta y Róger Umaña Chacón. Diagnóstico de la Expresión Escrita en la Educación Diversificada del Sistema Educativo Costarricense. Informe final. Instituto de Investigación para el Mejoramiento de la Educación Costarricense. 1986b.

Sánchez Avendaño, Carlos. "Historiografía de la enseñanza de la redacción en Costa Rica: los libros de texto" (por aparecer en Revista de Filología y Lingüística).

Sánchez Molina, Ana y Hazel Vargas Zeledón. Curso básico de redacción. San José: Ediciones Guayacán. 1990.

Sandoval de Fonseca, Virginia. Curso básico de redacción. San José: Editorial Fernández-Arce. 1970.

Sandoval de Fonseca, Virginia. Técnicas de redacción. Ciudad Universitaria Rodrigo Facio (sin editorial). 1978. 
Soto Alfaro, Carlos Luis, Benedicto Víquez Guzmán y Luis Fernando Ramírez Garro. Texto programado de ortografía española. San José: Editorial Nueva Década. 1983.

Varela Barboza Marubeni y Wálter Sandino Angulo. Redacción y ortografía: Una opción para todos. Heredia: Ediciones Marwal. 1993.

Vargas Araya, Aura Rosa y Virginia Sandoval de Fonseca. Castellano básico. La descripción. San José: Editorial Universidad Estatal a Distancia. 1978a.
Vargas Araya, Aura Rosa y Virginia Sandoval de Fonseca. Castellano básico. La narración. San José: Editorial Universidad Estatal a Distancia. 1978b.

Vargas Araya, Aura Rosa y Virginia Sandoval de Fonseca. Castellano básico. La exposición. San José: Editorial Universidad Estatal a Distancia. 1978c.

Vicenzi, Moisés. Metodología de la composición. San José: Imprenta Alsina. 1930.

Vicenzi, Moisés. La enseñanza de la puntuación. San José: Librería, Imprenta y Litografía Universal. 1939. 